\title{
Hepatocellular carcinoma statistics in South Korea
}

\author{
Young Eun Chon', Soung Won Jeong ${ }^{2}$, and Dae Won Jun ${ }^{3}$
}

${ }^{1}$ Department of Internal Medicine, CHA Bundang Medical Center, CHA University, Seongnam; 'Department of Internal Medicine, Soonchunhyang University College of Medicine, Seoul; ${ }^{3}$ Department of Internal Medicine, School of Medicine, Hanyang University, Seoul, Korea

\section{Incidence of HCC (2018)}

Incidence of HCC: Patients newly diagnosed with HCC from Korean National

Definition of HCC: International Classification of Diseases (ICD) code of C220 and rare incurable disease code of V193 (severe cancer)

standard population: population covered by health insurance in 2005

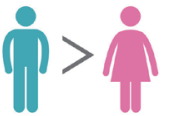

21.6 vs. 6.2

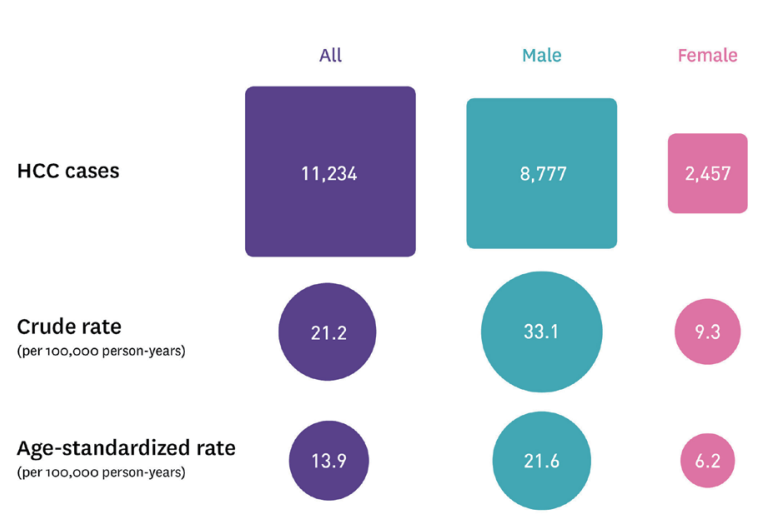

Changes in the crude incidence rate of $\mathrm{HCC}$ for the past 10 years
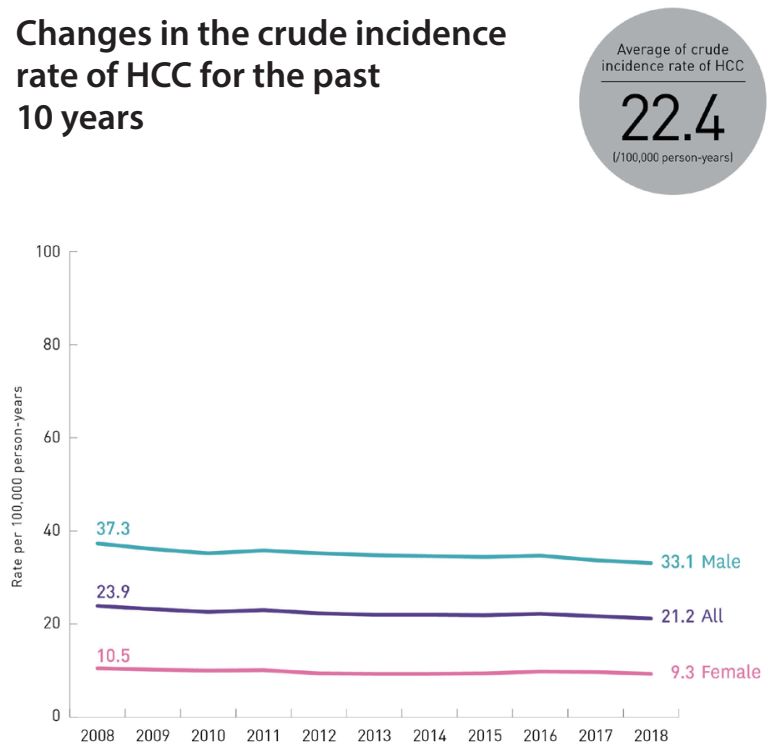

\section{Crude incidence rate of} HCC by age (2018) $60-79$

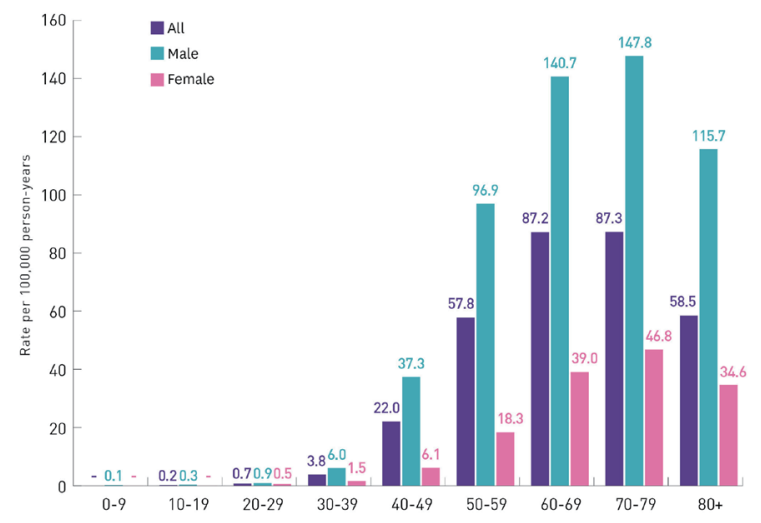

Changes in the crude incidence rate of $\mathrm{HCC}$ by age for the past 10 years
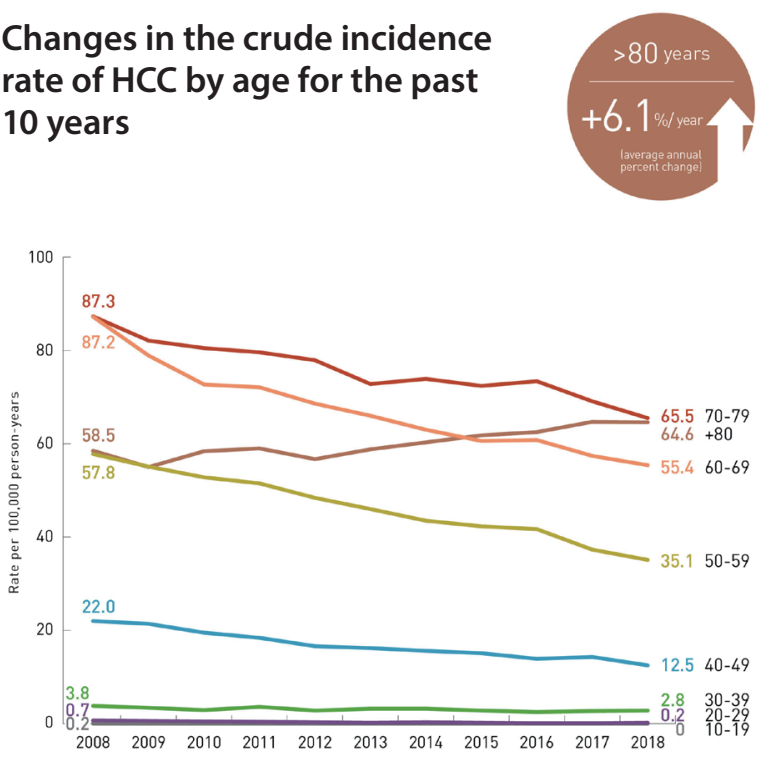

Copyright ( 2021 by Korean Association for the Study of the Liver

This is an Open Access article distributed under the terms of the Creative Commons Attribution Non-Commercial License (http://creativecommons.org/licenses/by-nc/3.0/) which permits unrestricted non-commercial use, distribution, and reproduction in any medium, provided the original work is properly cited. 


\section{Keywords: Crude incidence rate; Hepatocellular carcinoma; Incidence; South Korea}

Primary liver cancer is the sixth most common cancer worldwide and in South Korea (fifth in men and sixth in women), ${ }^{1,2}$ Hepatocellular carcinoma (HCC) accounts for the majority of primary liver cancer. ${ }^{3}$ Considering the high incidence and the poor prognosis of HCC, research on the epidemiology of HCC in South Korea is an important public health issue. ${ }^{4}$ The Korean Association for the Study of the Liver has recently published "2021 Hepatocellular Carcinoma Factsheet in Korea" to provide comprehensive information on HCC in South Korea, including the incidence, etiology, and comorbidities of HCC. ${ }^{5}$ Here in this Snapshot, we report the incidence of HCC in South Korea over the past 10 years, based on the data of "2021 Hepatocellular Carcinoma Factsheet in Korea."

The "2021 Hepatocellular Carcinoma Factsheet in Korea" used the data of HCC patients enrolled in the Korean National Health Insurance Service Database. HCC diagnosis was made when patients had both C220 (International Classification of Diseases code) and V193 (rare incurable disease code). In 2018, HCC was newly diagnosed in 11,234 patients $(8,777$ in male and 2,457 in female). A crude rate (CR) of HCC incidence was 21.2 per 100,000 person-years (33.1 per 100,000 person-years in male and 9.3 per 100,000 person-years in female). An age-standardized rate of HCC incidence was as 13.9 per 100,000 person-years (21.6 per 100,000 person-years in male and 6.2 per 100,000 person-years in female). According to the data from Statistics Korea, the CR of liver cancer incidence was 30.7 per 100,000 person-years (45.8 per 100,000 person-years in male and 15.6 per 100,000 personyears in female). ${ }^{2}$ The reason for the difference from our data is believed to be that the data of Statistics Korea included other types of liver cancer, such as intrahepatic cholangiocellular carcinoma (C221), hepatoblastoma (C222), and others. In 2018, stomach cancer had the highest incidence (CR, 57.1 per 100,000 person-years), followed by thyroid cancer ( 55.8 per 100,000 personyears) and lung cancer ( 55.8 per 100,000 person-years). Liver cancer ranked sixth (30.7 per 100,000 person-years), while prostate cancer ranked seventh with an incidence of 29.0 per 100,000 person-years, followed pancreatic cancer (14.8 per 100,000 person-years). Therefore, in addition to the CR incidence of liver cancer, the CR incidence of pure HCC of our study (21.2 per 100,000 person-years) was also higher than that of pancreatic cancer, which ranked eighth.

The CR of HCC incidence in 2018 was depicted according to age groups. The CR was the highest among patients aged $60-79$ years (87.2 per 100,000 person-years in the age 60-69 group and 87.3 per 100,000 person-years in the age $70-79$ group). The second and third highest incidence rate groups were patients aged $\geq 80$ years (115.7 per 100,000 person-years) and those aged 50-59 years (96.9 per 100,000 person-years), respectively. In patients under age 30, the CR was lower than 1 per 100,000 person-years. According to the 2018 Statistics Korea data, liver cancer was not in the top 5 for patients aged under 64 years, but it ranked fifth among patients older than 65 years after lung, colon, stomach, and prostate cancers, with the CR of 109.3 per 100,000 person-years. ${ }^{2}$ Compared to other types of cancer, HCC has a relatively high incidence rate in older patients.

\section{Abbreviations:}

CR, crude rate; HCC, hepatocellular carcinoma

\section{Corresponding author : Dae Won Jun}

Department of Internal Medicine, School of Medicine, Hanyang University, 222-1 Wangsimni-ro, Seongdong-gu, Seoul 04763, Korea Tel: +82-2-2290-8338, Fax: +82-2-2298-9183

E-mail: noshin@hanyang.ac.kr

https://orcid.org/0000-0002-2875-6139

\section{Soung Won Jeong}

Department of Internal Medicine, Soonchunhyang University College of Medicine, 59 Daesagwan-ro, Yongsan-gu, Seoul 04401, Korea Tel: +82-2-9419-1823, Fax: +82-2-709-9696

E-mail: jeongsw@schmc.ac.kr

https://orcid.org/0000-0003-2855-6011 
This Snapshot showed the changes in CR of HCC incidence over 10-year period (from 2008 to 2018). The CR of HCC incidence remained similar from 23.9 per 100,000 person-years in 2008 to 21.2 per 100,000 person-years in 2018, with the change being statistically insignificant (average percent change, $-0.94 \%$; $P=0.640$ ). The $C R$ of male patients was 37.3 per 100,000 personyears in 2008, and 33.1 per 100,000 person-years in 2018. The CR of female patients was 10.5 per 100,000 person-years in 2008 , and 9.3 per 100,000 person-years in 2018. Neither of the changes in $\mathrm{CR}$ during 10 years in both genders showed significant difference. Despite the decreasing trend in the incidence of HCC cases over the 10 years $(12,056$ in $2008 \rightarrow 11,234$ in 2018; average percent change, $-0.42 \% ; P<0.001)$, the maintenance of $C R$ may be due to the aging of the Korean society, which was more pronounced in the age group analysis. The changes in CR of HCC incidence during 10 years according to age groups were analyzed. In all age groups except for 80 years or older, the CR decreased from 2008 to 2018. However, in patients aged $\geq 80$ years, the $C R$ increased significantly over 10 years ( 58.5 per 100,000 personyears in $2008 \rightarrow 64.6$ per 100,000 person-years; average percent change, $1.4 \% ; P<0.001)$. The difference of HCC incidence among age groups and the increasing trends of HCC among old age seem to be consistent in other countries. In Taiwan (from 2003 to 2011), the incidence of HCC in patients over the age of 65 years significantly increased with an annual percentage change of $1.3 \%$, whereas HCC patients aged under 64 years showed a reducing trend of HCC incidence. ${ }^{6}$ In the United States from 1992 through 2015, the incidence of HCC decreased among younger and middle-aged adults regardless of sex, race, or ethnicity.

The HCC incidence in 2018 was the seventh highest among all cancer types in South Korea, and the crude incidence has not decreased over the past 10 years. Since HCC is often diagnosed at an advanced stage compared to other cancers, it tends to have a worse prognosis. Specifically, when HCC occurs in the elderly, it is more difficult to treat due to their frailty, and these patients become a part of the growing social burden in an aging society. Future strategies are needed to reduce the HCC incidence in the entire population of South Korea as well as in elderly patients, and to provide proper age-specific treatment for HCC patients.

\section{Authors' contributions}

Conception, Study design, Contribution to the data acquisition and statistical analysis, Writing papers: Young Eun Chon, Soung Won Jeong, and Dae Won Jun

\section{Acknowledgements}

This Snapshot used the data of "2021 Hepatocellular Carcinoma Factsheet in Korea", which was prepared by the Korean Association for the Study of the Liver (KASL) and the National Health Insurance Service (NHIS) based on MOU (grant no. NHIS-2021-1096).

\section{Conflicts of Interest}

The authors have no conflicts to disclose.

\section{REFERENCES}

1. Sung $H$, Ferlay J, Siegel RL, Laversanne M, Soerjomataram I, Jemal A, et al. Global cancer statistics 2020: GLOBOCAN estimates of incidence and mortality worldwide for 36 cancers in 185 countries. CA Cancer J Clin 2021;71:209-249.

2. National Cancer Center. Annual report of cancer statistics in Korea in 2018. Ilsan: National Cancer Center, 2018.

3. Villanueva A. Hepatocellular carcinoma. N Engl J Med 2019;380: 1450-1462.

4. Kim BH, Park JW. Epidemiology of liver cancer in South Korea. Clin Mol Hepatol 2018;24:1-9.

5. The Korean Association for the Study of the Liver. 2021 Hepatocellular Carcinoma Factsheet in Korea. The Korean Association for the Study of the Liver web site, <https://www.kasl.org/ bbs/?code=factsheet $>$. Accessed 23 Jun 2021.

6. Hung GY, Horng JL, Yen HJ, Lee CY, Lin LY. Changing incidence patterns of hepatocellular carcinoma among age groups in Taiwan. J Hepatol 2015;63:1390-1396.

7. Rich NE, Yopp AC, Singal AG, Murphy CC. Hepatocellular carcinoma incidence is decreasing among younger adults in the united states. Clin Gastroenterol Hepatol 2020;18:242-248.e5. 